\title{
1969 MEMBERSHIP DIRECTORY
}

AASKoV, RUTH L., Asst, Prof. of Fr., Lang. Lab. Dir., Augsburg College, Minneapolis, Min. 55404

ACQUISITIONS LIBRARIAN, North Texas State Univ., Library Dept., Denton, Texas 76203

ADAM, RICHARD, Dir. Lang. Lab., Western Reserve Academy, Hudson, Ohio 44236

ADAMS, KENNETH R., For. Lang. Dept., Weber State College, Ogden, Utah 84403

ADAMS, MRS. SUDIE M., Asst. Prof. of Sp. and Port., Baylor Univ., Box 159, Waco, Texas 76703

ADORNO, MRS. ROLENA K., Dir. Lang. Lab, Univ. of Hartford, 200 Bloomfield Ave.. Hartford, Conn. 06117

AGOSTINO, MISS NELLIE, 777 Burnside Ave., East Hattford, Conn. 06117

AGUIRRE, A. A., Dir. Lang. Lab, Indiana Univ., South Bend, Northside Blvd., South Bend. Ind. 46615

AGUIRRE, MRS. OLGA, Dir. Lang. Lab., Stratford College, Danville, Vir. 24541

AHERN, MR. JOHN A., Westerchseter Community College, 75 Grasslands Rd., Valhalla, N. Y. 10595

AHRENS, MISS CHRISTA, Dir. For. Lang. Labs, Georgia State College, 33 Gilmet St., S. E. Box 415, Atlanta, Georgia 30303

AIKENS, H. F., Dir. Lang. Lab, Dalhonsie University, Halifax Nova Scotia, CANADA

ALAMEDDINE, FAROUK B., 20023 Trinity Ave., Detroit, Michigan 48219

ALEMANY, GILBERTO R., Instr. Spanish, Ricker College, Houlton, Me. 04730

ALIOTTI, VIRGINIA BROWN. Dir. Lang. Lab. Madison College, Box X, Hatrisonburg, Va. 22801

ALLEN, DR. HAL., Assoc. Prof. Ger., Hendrix College, Conway, Arkansas 72032

ALLEN, ROBERT, Dit. Lang. Lab., Rutgers University, 508 Newark Ave., Piscataway, N. J. 08854

ALTMAN, LEON, San Francisco State College 1600 Holloway, San Francisco, Cal. 94132

AMBERT, GARY JOHN, Dir. Lang. Lab, East Carolina Univ, Greenville, North Carolina 27834 ANDRETZ, SERGEY N., Dir. Lang. Lab. Michigan State Univ. A126 Weells Hall, East Lansing. Michigan 48823

ANNABle, PATRICIA C., Chmn. Slippety Rock State College, Slippery Rock. Penn. 16037 ARANITI, NAZMI, Dir Lang. Lab, Quinnipiac College, 303 Noble St. West Haven, Conn. ARBAIZA, N. D., Chmn., Lang. Dept., Rockland Community College, 145 College Rd. Suffeta. N. Y. 10901

ARENDT, J., For. Lang. Conslt., 3709 48th Ave. S., Minneapolis, Minn. 35406

ARmstrong, JoANne, Assoc. Prof., Univ, of Texas at El Paso, Box 5, El Paso, Texas 79902 ARNOLD, KATHRYN, Dir. Lang. Lab. Adelphi Univ., Garden City, N. Y. 11530

ASSELIN, JEAN-GUY, Technician, Carleton Univ., Lang. Lab. Colonelby Drive, Ottawz 1. Ontario CANADA

ATKINSON, MRS. GEORGE M., 35361/2 Asbury Ave., Dallas, Texas 75205

ATTIG, MR. CECIL, 800 North State College Blvd., Fullerton, Cal. 92631.

AUGER, GILES E., Asst. Prof. Masson College, Springvale, Maine 04083

AUGUSTA, SISTer MARY, Prof. of Fr. and Sp., Xavier Univ, of Louisiana, Pine and Palmetto Streets New Orleans, Louisiana 70125

AUSTIN, ROBERT P., Lang. Lab. Dir. Loyola Academy, 1100 N. Laramie, Wilmette, Ill. 60091

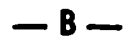

BAAK, DR. L. E., Chmn. Dept. Mod. Lang., Morningside College, Sioux City, Io. 51106 BAIMAS, MR. JOHN C., Head, Lang. Dept., Fitchburgh, Ma. 01420 BAKER, REID E., 80 East 14th Ave., Apt X. Columbus, Ohio 43201

BAKER, ROBERT L., Chmn. Dept. of Rus., Middlebury College, Middlebury, Vt. 05753

BALCAEN, HUBERT, 500 Dysart Road. Winnepeg 19. Manitoba, CANADA 


\section{Membership Directory}

BALDWIN, MrS. VIRGINIA, Cbmn, For. Lang. Crystal Lake Community H. S., Franklin. St., Crystal Lake, III. 60014

BALl, MRS. JeanNiE, Chmn, Fr. Dept., Madeira School, Greenway, Va. 22067

BALTINS, VIVISA N., Saint Francis College, Fort Wayne, Ind. 46808

BARGER, KENNETH S., Dir. Lang. Lab., P. O. Box 1, Columbia, III. 62236

BARON, MRS. GAIL, Academic Coord., Brothers College, Drew Univ., Madison, N. J. 07940 BARR, CHARLeS J., Dir. Lang. Lab., St. John's College, 5118 Seminary Rd., Camarille, Cal. 93010

BARRETT, J. E., Head of Language Department. St. George's School, W. 2929 Waikiki Road, Spokane, Washington 99208

BARTH, REV. GILBERT, T. O. R., Dir. Dept. of Lang., The College of Steubenville, Franciscan Way, Steubenville, Ohio 43952

BATTO, MRS. TERESA, Dir. Lang. Lab. Loyola College, 4501 North Charles St., Baltimore. Md. 21210

BAUdIN, PHILIP R., Coord. FL. Hamline Public Schools, P. O. Box 100 Seattle, Wash. 98166 BAXA, PAULINE, Dean Junior College, Franklin, Mass 02038

BAUMLIN, MLLE., Milton Academy, 325 Randolph Ave., Milton, Ma 02186

BAYNARD, MRS. ANTHONY, Chmn. French Dept., Maumee Valley Country Day Scbool, 1715 S. Reynolds Rd., Toledo, Ohio 43614

BEeCHER, MRS. JEAN, Moravian College, Bethlehem, Penn. 18018

BELl, ELIZABETH, Lang. Lab. Dir., Box 348, Allen Univ., Columbia, S. C. 29204

BELl, KeITH R., Prof. of Mod. Lang., Mid-American Nazarene College, 2030 College Way. Olathe, Kansas 66061

BenBOW, CAROLYN, Lang. Lab. Dir., Teachers' College, Columbia Univ., Box 66, 525 Wer 120 th New York, N. Y. 10027

BENOIST, SR. GLORIA, Lang. Lab. Dir.. St. Mary's Dominican College 7214 St. Charles Ave. New Orleans, Louisiana 70118

BERNIER, HENRI, Walsh College, 2020 Easton Street, Canton, Ohio 44720

BERNSTEIN, JAY, Language Advisor, Nassau Community College, 79 Constable Lane, Levit• town, N. Y. 11756

BERTAUX, ALAN, Asst. Dir., 2105 Hawthorne Ave., Elkridge, Md. 21227

BIBEAU. GILLES, Chef Des Laboratories de Langucs, Universite de Montreal (Faculte des Lettres) C. P. 6128, Montreal 3 Quebec CANADA

BISSON, PAUl R., Greenfield Community College, 214 Federal St., Greenfield, Mass. 01301

BLAIR, JOSEPH C., Dir. Lang. Lab, Language Lab $A$ and $S$ 39, Univ. of Mo., Columbia, Mo. 65201

BLOSJO, R. V., Asst. Prof., Alderson-Broaddus College, Philippi, W. Va. 26416

BLOUNT, JAMES P., Mod. Lang. Lab, College of William and Mary, Williamsburg, Va. 2318s

BOARD OF EDUCATION, Freehold Regional H. S. Dist., 225 Schank Rd., Freehold, N. J. 07728

BOBETSKY, PROF. V., Dir. Lang. Lab, Polytechnic Inst. of Brooklyn, 333 Jay St., Brooklyn. N. Y. 11201

BODNAREK, MRS. VIRA. Dir. Lang. Lab. 3939 W. 79th St. Chicago, III. 60632

BOGART, WILlIAM F., 123 North Williamson Rd., Blossburg, Penn 16912

BOHN, JERRY, Asst. Dir., Macalester College, St. Paul, Minn. 55104

BOLDUC, MARCEI, Hd. of French, Acad. Dean, Oblate College and Seminary, 149 Woodland St. Natick, Ma 01760

BOOTH, EDW ARD F., Consle., Mod. For. Lang., State Dept. of Educ., Augusta, Me. 04330

BOOTHE, ROBERT O.. Cal State Polytechnic College, San Luis Obispo, Cal. 93401

BORN, WARREN C., Dir. For. Lang. Educ., N. Syracuse Central Schools, 305 S. Main St.. North Syracuse, N. Y. 13212

BOSW AU, HERBERT, Box 8198 Univ. Station, Grand Forks, N. D. 58201

BOUGARD, ROGER. Language Laboratory Director. 14061/2 Claredon M.. Durham. North Carolina 27705

BOUNDS, MRS. MURIEL, Teacher, Hermosa Junior High, 906 N. Butler, Framington, N. M. 87401

BOYDEN, PATRICK C., Kent State University, Kent, Ohio 44240 
BRADSHAW, M. E., 4431 Durer Parkway, Sacramento, Cal. 95823

BRADY, NORMA, Inter-American University, Box 1293, Hato Rey, Puerto Rico 00919

BRATNOBER, H. L. JR., For. Lang. Pro., Southwest Minnesota State College, Marshall, Minn. 56258

BRENDAN, SISTER ROSE, O. P., Barry College, N. E. Second Ave., Miami, Florida 33161

BRENTON, MRS. V., Dir. Lang. Lab. Vincennes Univ. Junior College, Vincennes, Ind. 47591 BREYMAN, MRS. GABYRON B., Chm. French, Cal. Lutheran College, Thousand Oaks, Cal. 91360

BRIGOLA, DR. ALFREDO, Coord., For. Lang. Dept., F. I. Lab Dit., Univ. of Redlands, Redlands, California 92373

BRINK, SISTER M. CAROLYN, RSM, Our Lady of Cincinnati College, Edgecliff, Walnut Hills, Cincinnati, Ohio 45206

BROSSMAN, ERIKA S., Asst. Dir. Lang. Lab., Albright College, Reading, Pa. 19604

BROSSMAN, DR. LUTHER F., Dir. Lang. Lab. Albright College, Reading, Pa. 19604

BROWN, ELDON I., Springfield College, 1500 North Fifth Street, Springfield, Ill. 62702

BROWN, MRS. FLORENCE G., Chief, Acad. Support Sec., Defense Lang. Inst. Support Com. mand 5312 Salisbury, El Paso, Texas 79924

BROWN, C. P., Foreign Language Dept., Western Kentucky University, Bowling Green, Kentucky 42101

BROWN, S. W., Professor Mod. Lang., Knox College, Galesburg, Ill. 61401

BSHARAR, SISTER M. ALBAN. Dir. Lang. Lab. College of New Rochelle, New Rochelle, N. Y. 10801

BUCK, DR. GEORGE C.. Dir. Lang. Lab, Univ, of Washington, 118 Denny Hall, Seattle, Wash. 98105

BUNDY, BONNIE, Dir. For. Lang. Lab., The Defiance College, C/O L. C. Olds, Defiance, Ohio 43512

BURR, YOLANDA, Lang. Lab. Supv. Alfred Univ., R. D. Ho. 3, Wellsville, N. Y. 14895

BUTEAU, MAGDELHAYNE, 3465 Durocher Street, Montreal, Quebec, CANADA

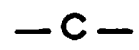

CallanaN, James C., Dir. Lang. Lab, Southern Illinois Univ., Edwardsville, Ill. 62025

CAMPA, DR. ROMAN DE LA, Chmn. Lang. Dept., Briar Cliff College, Sioux City, Iowa 51104 CAPRETZ, PIERRE J., Dir. Lang. Lab., 111 Grove St., New Haven Conn. 06510

CARR, EMORY W.. Instr., P. O. Box 114, Marshall Univ., Huntington, W. Va. 25701

CARTIER, F. A., Dir. of Development, Defense Language Institute, English Language School. 7415 Buckskin, San Antonio, Texas 78227

CARTWRIGHT, RICHARD, Chmn. Mod. Lang. Dept., Mt. Hermon School, Me. Hermon, Ma 01354

CERVIZZI, MRS. CHRISTINE E., Northbridge H. S., Whitinsville, Mass. 01588

CHERRY, RALPH, Lang. Lab. Dir., Southeastern State College, Durant, Oklahoma 74701

CHOMAR, DR. ORLANDO, Dir. Lang. Lab, College of Mount St. Joseph on the Ohio, 5701 Delhi Rd., Mt. St. Joseph, Ohio 45051

CHOMEI, MISS TOSHIKO, Instructor, Ochanomizu University, Otsuka 2 Chome, Bunkyo-ku, Tokyo, JAPAN

CHRISTMAN, HENRY R., Instr., 215 Wellington Ave., Lincoln Park, Reading, Pa, 19600

CIBECCHIA, DR. FRANCESCA, Dir. Lang. Lab, Duquesne Univ., Pittsbungh, Penn. 15219

CICERO, A. E., Assoc. Prof., 808 Ridge Avenue, Pittsburgh, Penn. 15212

CLARK, BEN T., Coord. of Lang Studies, Univ. of Cal., Santa Cruz, Merrill College, U. C. S. C. Santa Cruz, Cal. 95060

CLARK, EDWARD P., Foreign Languages, Moorhead State College, Moorhead, Mn. 56560

ClaUdel, CALVIN A., Box 98, West Virginia Wesleyan College, Buckhannon, W. Va. 26201

COFFIN, MRS. T. C., Lang. Lab Asst., The Baldwin School, Bryn Mawr, Pa. 19010

COLECCHIA, DR. FRANCESCA, Director, Language Laboratory, Duquesne University. Pittsburgh, Pennsylvania 15219

COLL, MISS PAULINE, Chmn. For. Lang. 135 Mulberry Rd., Danville. Va. 24541 
COLlins, RalPH S., Chmn. Lang. Dept., Maryland College, 1741 Linda Lane, Maryville. Tenn. 37801

COLOMBO, MRS. RUTH W., Hd., Lang. Lab. Dept., Natick H. S., 15 West Street, Natick. Ma. 01760

CONKEY, MRS. JEAN S., Supv. Lang. Lab., Wheaton College, Norton, Mass. 02766

CONWELl, DR. MARILYN J., Valley View Apts., 251 West DeKalb Pike, King of Prussia, Penn. 19406

COOK, MARGaret A., Dit. Lang. Lab, Downtown Campus, Indiana Univ. at Indianapolis, 222 East Michigan St., Indianapolis, Ind. 46204

COON, REVEREND DAVID P.. Assist. Headmaster, Iolani School, 563 Kamoku St., Honolulu. Hawaii 96814

COOPER, DR. D. CHAVY, Chairman, Lang. Dept., Keuka College, Keuka Park, N. Y. 14478 COOPER, HENRY F., Dit. Lang. Lab, University of Oregon-Romance Langs., Eugene, Oregon 97403

CORPACI, JOHN, Chmn., Lang. Dept., Cheshire Academy, Box 306 Cheshite, Ct. 06410

CORRY, BROTHER MICHAEL, Christian Brothers Academy, Lincroft, N. J. 07738

CORTES, ELADIO, Lang. Dir., Ruggers Univ., Camden, N. J. 08102

COSMaN, MRS. T., Dir. Lang, Lab, Barnard College, 11 Lehman Hall, 26 Claremont Ave., New York, N. Y. 10027

COSTA, RONALD KENT, Dir. Lang. Lab. Mass. State College at Farmington, 20 Greenwood St. Oak Hill, Newton, Mass. 02159

COTE, NORMaN R., Head For. Lang. Dept.. Notth Shore Community College, 3 Essex Street. Beverly, Mass. 01915

COTE, PAUL E., Dir. Lang. Lab., Avon H. S., Avon Conn. 06001

COURNOYER, MISS MADELEINE A., Chairman Foreign Language, Proviso Township High Schools, Proviso East High School. First Avenue and Madison, Maywood, Illinois 60153

COURTOIS, NELlY, Newton College of the Sacred Heart. 885 Centre St., Newton 59, Newton, Ma 02159

COX, SHIRLEY O., Chmn. Mod. Lang. Edison Junior College, Cypress Lake Drive, Fort Myers. Fla. 33901

COYNE, MARTIN J., Techn. Asst. Lang, Lab, Univ. of Massachusetts, Boston, 100 Arlington St., Boston, Mass. 02116

CRAIG, MRS. RUTH PARle. Dir. For. Lang. Lab, Chmn For. Lang., Santa Rose Junior College, Santa Rose, Calif. 94501

CRAIGHILl, MARY NUTE, Harvard Univ., Boylston Hall, Harvard Yard, Cambridge, Mass. 02138

CREAMER, JOHN F., Dir. Ll, Hudson H. S., Cottage Street, Hudson, Mass. 01749

CRIMINALE, LEONARD R., Dir. Lang. Lab., Elmira College, Elmira, N. Y. 14901

CROWNER, DR. DAVID, Dir. Lang. Lab., Gettysburg, Penn. 17325

CRUZ, Carlos M., Supv. Lang. Lab., Univ. of Rochester, River Campus Station, Rochester. N. Y. 14627

CUNEO, WILliAM J., Dir. Lang. Lab., Holyoke Community College, 170 Sargeant Street. Holyoke, Mass. 01040

CUNNINGHaM. M. J., JR., Chmn. Lang. Dept. New Mexico Junior College, Lovington, Higbway, Hobbs, N. M. 88240

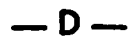

DACEY, D. P., KH 316, 1300 Elmwood Avenue, Buffalo, New York 14222

DALGO, RODNEY C., Dir. Lang. Lab, University of South Carolina, Columbus, S. C. 29208

DARRAS, MiChael, Assoc. Prof. of French. Memorial Univ, of Newfoundland, St. John's Newfoundland, CANADA

DAY, STEPHEN, Queen's University Kingston, Ontario CANADA

DEBONE, DR. G., Head of Lang. Dept., LaGrange College, LaGrange, Georgia 30240

DELANEY, JOHN, Wolcott H. S., Wolcott, Conn. 06716

DELANOEYE, MRS. JOHN, Dh, Foreign Lang. Lab, California State College at Los Angeles. S151 State College Drive, Los Angeles, Cal. 90032

DENNIS, LARRY KENT. Dir. Instructional Media. Concordia College, Moothead, Minn. 56560 
DE PAUL, SISTER MARIE, Chmn. Lang. Dept., Bishop Gallagher, 19360 Harpet Ave., Harper Woods, Michigan 48236

DePeauX, ReV. R. C., Chmn., Lang. Dept., St. Norbert College, West De Pere, Wis, 54178 DEPT. OF MOD. LANG., Boston Univ., 236 Bay State Rd., Boston, Mass. 02215

DESAlVO, BeNNIE T., 50 South Main Strect, West Hartford, Conn. 06100

DETI, DANIEL, Chmn. Lang. Dept., College of the Desert, Palm Desert, Cal. 92260

DETORRE, MR. EMILIO, Manhattanville College, Purchase Strect, Harrison, N. Y. 15077

DeVARGaS, DIEGO, FL Coord., Carlsbad Municipal Schools, 103 West Hagerman St., Carlsbad, N. M. 88220

DEVliN, JUSTINE M., Hd. Lang. Dept., Lawrence H. S., Lawrence, Mass. 01841

DICKERMAN, WilliaM C., Dir. Lang. Lab, 3801 Cullen Boulevard, Houston, Texas 77004

DIETRICH, ELMER, The Miller Public Schools, Miller, South Dakotz 57362

DIRECTOR, ENGLISH DEPARTMENT, Faculty of General Studies, University of Puerto Rico, Rio Piedras, Puerto Rico

DIRECTOR, FOREIGN LANGUAGE DEPARTMENT, Sam Houston State College. Huntsville, Texas 77340

DIVINCENZO, vito J., Villanova Univ., Spring and Hilldale Rd., Malvern Rd. 2, Pena. 19455

DIXON, FRANCE P., Language Laboratory Director, MeKendree College, Lebanon, Illinois 62254

DODDY, MICHAEL, S., 190 West 168th St., Bronx, N. Y. 10452

DODGE, JAMES W., Tech. Dir. Lang. Lab, Brown University, Box E. Providence, R. I. 02912 DONATELLI, JOSEPH, Dir. Lang. Lab, University of Akron, Akron, Ohio 44304

DOVER, HUGH C., 508 North Buchanan Blvd., Durham, N. C. 27701

DOWNING, MRS. B., West Texas State University, Canyon, Texas 79015

DRAGONE, DR. O., Dir. A. I. C., State Street, Springfield, Mass, 01109

DRUMMOND, DONALD L., Audio-Visual Coord., San Antonio College, 1001 Howatd St., San Antonio, Texas 78212

DUCHARME, SISTER M. J., Chmn. Mod. Lang, River College, Mashua, N. H. 03060

DUCRETET, MR. PIERRE, University College 213, Univ, of Toronto, Totonto 3, Ontatio CANADA

DUNBAR, DR. H. B., Chairman, Division of Liberal Arts, New Yosk City of Community College, 300 Jay Street, Brooklyn, N. Y. 11201

DURDEN, JOHN D., 4738 Everhart Dr., North Canton, Ohio 44720

DUVAL, F. A., Chmn. Dept. of Classical and Mod. Lang., Cornell College, 710 8th Ave. N.. Mt. Vernon, Iowa $\$ 2314$

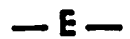

ECONOmos, R. M., Prof., Pace College, 41 Park Row, New York, N. Y. 10038

EDIE, MRS. JOHN, Chmn. French Dept., Dir. Lang. Lab Northrop Collegiate School, 311 Kenwood Pkwy, Minneapolis, Minn. 35403

EDLINE, ClAUDE, Dir., Language Lab. Gilman School Inc., 5407 Roland Ave., Baltimore, Md. 21210

EDWARDS, DAVID WaYNE, Dir. Lang. Lab, Emory Univ., Dept. of Romance Lang., Atlanta, Ga. 30300

EISENSTADT, RALPH, West Chester State College, West Chester, Penn. 09380

ELDON, MRS. JANE R., Dir. Lang. Lab, North Central College, Naperville, Ill. 60540

ELGUEZABAL, MR. E., Loyola Univ., P. O. Box 81, New Orleans, La. 70118

ELLIS, BARRY L., Teacher, 28500 Avondale Ave., Inkster, Mich. 48141

ELMENDORF, WILLIAM E., Dir. Lang. Lab, Western Washington State College, Belfingham. Wash. 98225

ELMORE, ROGER J., Acting Head, Mod. For. Lang., Cumbetland College, Box 121, Cumber. land College Station, Williamsburg, Ky. 40769

ELTON, MAURICE G., Southern Methodist Univ., Clements Hall, Dallas, Texas 75222

EMERY, EDMOND L., Chmn. Lang. Dept., Milford Academy, 150 Gulf St., Milford, Conn. 06460

EMILY, SISTER MARY, Fr. Instr. Lab. Dir., Mount Mercy College, 1330 Elmburst Drive N. E., Cedar Rapids, Io. $\$ 2402$ 
EPPINK, ALICE J., Lib., Center for Applied Linguistics, 1717 Massachusetes Ave., N. W., Washington, D. C. 20036

ERICKSON, PROFESSOR C. T., Div. Lang. Lab, S. U. N. Y., Dept. of Romance Lans.. Stony Brook, N. Y. 11790

ESPINOSA, LORENZO, Language Lab Supervisor, University of California, Riverside, California 92502

ESSA, DR. ROBERT, Chairman Foreign Language Dept., Sierra College, 5000 Rocklin Road, Rocklin, California 95677

EWALD, WILlIAM, Lang Lab Dir., Box 9-C. Concordia College, River Forest, III. 60305

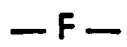

FAIRLEY, MRS. MAMIE, Hd., For. Lang., Magnolia High School, Box 428, Moss Point, Miss. 39563

FANG, ILSE M., Prof., Suffolk University, 41 Temple St., Boston, Mass. 02114

FANTINI, ALVINO E., Dir., For. Lang. Office, Experiment in International Living, Kipling Rd., Brattleboro, Vt. 05301

FAULKNER, JAMES C., Division Chmn., 17 Cedar Ridge Rd., New Paltz, N. Y., 12561

FAYE, MARIO R., Tacoma Washington Community College, Tacoma, Washington 98463

FEINBERG, ROSA CASTRO, Rober E. Lee Jr., H. S. 3100 N. W. 5th Ave., Miami, Fla. 33127

FELSHER, DR. WILlIAM M., Assoc. Prof., Box 375B, Cullowhee, N. C. 28723

FENNER, DR. REST JR., Hd. Lang. Dept., 91 South Orange Ave., Livingston, N. J. 07039

FERGUSON, LOU, Chmn. FL Dept., Glenbard East High School, 1014 S. Main Street, Lombard, III 60418

FERLAND, MR. MARCIEN, Dir. Lang. Lab, Univ. of Winnipeg, Winnipeg, Manitoba, CANADA

FERNANDEZ, DR. AUGUSTINE, Asst, Prof. Romance Lang., Bishop College 3837 Simpson Stuart, Dallis, Texas 75241

FERSEN, MRS. NINA, Williams College, Williamstown, Mass. 01267

FINK, KARL T., Instr., Luther College, 811 Iowa Avenue, Decorah, Iowa 52101

FISHER, RONALD D., LL Dir., La Sale College, 20th St., At Olney Ave.. Philadelphia, Penn. 19141

FITCH, C. BRUSE, Dir., Lang. Lab, Transylvania College, 300 Nortb Broadway, Lexington, Ky. 40508

FLeURANT, R., Pine Crest School, 1501 N. E. 62nd St., Fort Lauderdale, Fla. 33308

FORD, ANTHONY F., Dir. Lang. Lab, 360 Huntington Ave., Boston, Mass. 02115

FOREIGN LANGUAGE DEPARTMENT, Box XL, Alaska Methodist Univ., Anchorage, Alaska 99504

FORTNER, DEBORAH, Lang. Dir., Citrus H. S., Inverness, Fla, 32650

FOURNET, JAMES F., 1004 South Oak Street, Hammond, La. 70401

FOURNIER, ROBERT, For. Lang. Conslt., 17 Webster St., Suncook, N. H. 03275

FOWLER, MISS BETTY, Coord. Const. in FL, Dept. of Education, Stanislaus County Schools. P. O. Box 1697, Modesto, California 95354

FRANKS, MRS. ANITA W.. Spanish Teacher, Thayer Academy, 745 Washington St., Braintree, Mass. 02185

FRAYNE, MRS. EVA, Lang. Lab Coord., 2 Main Street, Champaign, Ill. 61820

FRAZIER, ESTELLE C., French Teacher, Academy Heights H. S., P. O. Box 1210, Pinehurst, N. C. 28374

FRECHETTE, DR. ERNEST A., Dept. of For. Lang. Educ., Florida State Univ., Tallabassee, Fla. 32304

FRIEDMAN, FRANK M., 1401 East Court St., Flint, Mich. 48503

FROST, ARTHUR F., Dir. Lang. Lab, Princeton Univ., 304 E. Pyne Bldg., Ptinceton, N. J. 08540

FUGATE, JOE K., Kalamazoo College, Kalamazoo, Michigan 49001

FUSCO, JOSEPH F., Laboratory Director. Dept. of English for Foreign Students. St. Michael's College, Winooski, Vermont 15404 


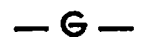

GAGON, GILles, Royal Military College of Canada, Massey Bldg., Kingston, Ontario CANADA

GALVIN, THOMAS F., Dir. For. Lang., Marshfield Public Schools, Marshfield, Mass. 02050

GARFINKEL, ALAN, 154 North Oval Drive (Derby 116), Columbus, Ohio 43210

GARNeR, MR. GARY N., Louisiana College, Pineville, Louisiana 71360

GATCHELL, MRS. ALICE, 470 East Luckwood, Webster Groves, Mo. 63119

GARRISON, H. LESLIE, Hd., Dept. of Romance Lang., Trinity Junior College, Langley, British Columbia CANADA

GASCHLER, MICHAEL, Instr. of Ger. Grand View College, Des Moines, Iowa 50313

GAUDIN, LOIS S. Prof., Brooklyn College of Cuny, Dept. of Mod. Lang., Brooklyn, N. Y. 11210

GAUTIER, ROGER, University of Saskatchewan, Saskatoon, Saskatchewan CANADA

GefFert, CAROL W., Assoc. Prof. of Ger., St. Andrew's Presbyterian College, Laurinburg. N. C. 28352

GeIGer, RICHARD A., Asst. Prof., Franklin and Marshall College, Lancaster, Penn. 17604

GELINEAU, MRS. ANNE H., G.4 Boylston Hall, Harvard Yard, Cambridge, Mass. 02138

GENDRON, RAYMOND, Hd. Lang. Dept. Seekonk Senior H. S., Seekonk, Mass. 02771

GIANNeTti, GeORGE. Chmn. Fl, Oak Park H. S., 13701 Oak Park Blvd., Oak Park, Mich. 48237

GIFFORD, NATHANIEL H.. Brooks School, North Andover, Mass. 01845

GILBERT, MRS. SUSI, North Church St., Spartanburg, S. C. 29301

GIONET, BROTHER DENIS, Dir. Lang. Lab. Scolasticat Central, 7000 Marie-Victorin Montreal, CANADA 462

GLENNIE, MRS. VIOLA, Instr., Alpena Community College, 666 Johnson St., Alpena, Mich. 49707GlODell, MARCUS, Prof. Spanish, 251 Belmont St., Worcester, Mass. 01605

GOETZ, DONALD R., Dir. Lang. Lab., Lincoln H. S., 1433 South 8th St., Manitowoc, Wis. 54220

GOLDSTeIN. E. M.. Assoc. Prof., Univ, of Ottawa, Dept. of Ling., 165 Waller St., Ottawa 2. Ontario CANADA

GOMEZ. FERNANDO. Ridgewood High School, 627 East Ridgewood Avenue, Ridgewood, New Jersey 07451

GONZALES. MISS ANGelitA FLORO, California State College at Hayward, 23976 Second St.. Apt. 9, Hayward. California 94541

GOODYEAR, STEPHEN, Hd. For. Lang. Dept., Hull H. S., 180 Main St., Hull, Mass. 02045

GORDON, IAN B., LL Dir., Baptist College at Charleston, P. O. Box 10087, Charleston, S. C. 29411

GOULOT, SR. NOEllA. Notre Dame College, 2321 Elms St., Manchester, N. H. 03104

GOYER, DOREEN S., Asst. Prof., Mod. Lang. Dept., Southwest Texas State College, San Marcos, Texas 78666

GRAMMATICOFF, ALEX, 3400 Pershall Rd., St. Louis, Mo. 63135

GRAY, MRS. MARY C., P. O. Box 279, Lenoir Rhyne Station, Hickory, N. C. 28601

GREEN VALE SCHOOL, Glen Head, Long Island, New York, N. Y. 11545

GREEN, JACK BYRON. Dir. Lang. Lab. Modeste Junior College, Modesto, Cal. 95350

GREEN, ROBERT B., Junior. Dir. Lang. Lab, 48 Smithfield St., Buckhannon, W. Va. 26201

GREENE, MRS. ESTELlE S., Dir. LL, Rutherford Fairleigh Dickinson Univ., 207 Montress Ave.. Rutherford. N. J. 07070

GREGORY, ClARA, Conslt.. Texas Education Agency, 1202 Newning, Austin, Texas 78704

GRIFFIN, REV. J. J.. Dir.. Audio-Visual Lab, Allentown College, Center Valley, Penn. 18034 GRILlO, PAUl, Head, Firench Department. North Country School, Lake Placid, New York 12946

GRITTNER, FRANK, State Supv. of Mod. For. Lang., 710 Woodward Dr., Madison, Wis. 53704

GROVER, SISTER M. ANSELM. 3500 Mountain Blvd., Oakland, Cal. 94619

GRUNDSTROM, ALlaN W., Asst. Prof. French, Bucknell University, Lewisburg, Penn. 17837

GUENETTE, MR. PAUL. G., Dir. For. Lang., Town of Brookline. Town Hall, 333 Wash. St., Brookline. Mass. 06146 
GUNDlaCH, C. M., Supv. Lang. Lab, Wilmington College, Box 990 College Post Office, Wilmington, Ohio 45177

GURA, ROSALYN, Lang. Lab. Supv., Youngstown State Univ, 410 Wick Ave., Youngstown, Ohio 44503

GUZEK, MISS KATHLEEN J., French Teacher, Ontonagon Area High School, 304 Pennsylvania Avenue, Ontonagon, Michigan 49953

guzman, ERNesto a., Coord., For. Lang., Tarrant Co. Junior College, South Campus, Ft. Worth, Texas 76119

\section{$-\mathrm{H}-$}

halem, hilmanN voN, Head Lang. Div., Getman Center Boston, 170 Beacon St., Boston. Mass. 02116

HAGEN, MRS. DONALD F., Dir. Lang. Lab, Fairleigh Dickinson Univ., Florham-Madison Campus, 285 Madison Ave., Madison, N. J. 07940

HALI, LEWIS, Academic Dean, Lees-McRae College, Banner Elk, North Carolina 28604

HALENZ, ESTER, Chmn. Mod. Lang., Columbia Union College, Takoma Park, Maryland 20012

HAMMOND, MR. JOHN H., Dept. of For. Lang., Texas Christian Univ., Fort Worth, Texas 76129

HAMSON, ERWIN M., Dir. Lang. Lab, 1401 Mason Hall, Ann Arbor, Mich., 48104

HAND, RAYMOND, Alexander City State Junior College, Alexander City, Alabama 35010

HANSON, DAVID A., Asst. Prof. Russ., German and Slavic Lang., Brandeis Univ., Waltham, Mass. 02154

HARDING, MRS. JACOBINA, LL Supv., Alamo Heights H. S., 6900 Broadway, San Antonio. Texas 78209

HARRISON, BROTHER EDW ARD, 2841 de Lorimier, Ste-Foy, P. Quebec, CANADA

HARRISON, C. NORRES, Washington College, Chestertown, Maryland 21620

haRTMAN, ALICE, Chmn. Lang. Dept., 401 North Latkin, Joliet, III. 60433

HASENCLEVER, Lang. Faculty, Bennington College, Bennington, Vt. 05201

HAWKINS, PAUL, JR., Acting German Chairman, West Virginia Univ., Parkersburg Center, 1110 16th St., Vienna, W. Va. 26101

HAYASE, MISS YURIKO, Director Language Laboratory, Winthrop College, Rock Hill, South Carolina 29730

HAYWARD, EARL F., Lang. Lab Dir., Alma College, Alma, Mich. 48801

HEADQUARTERS, DLIWC Academic Libtary, Bldg. 302, Presidio of Monterey, Cal. 93940

heERMANS, LILIIAN, Dir. Lang. Lab, Vassar College, Poughkeepsie, N. Y. 12600

HEGGOY, KORE N., Dir. Lang. Lab, Randolph-Macon College, P. O. Box 726, Ashland,.Va. 23005

HEIMAN, MRS. MONICA, West Texas State Univ. Canyon, Texas 79015

HEINE, HEINRICH, 3004 Woodlawn Ave., Falls Church, Va. 22042

HEPBURN, ERIC, Chmn. For. Lang., 335 Wadsworth Ave., New York, N. Y. 10040

HERNANDEZ, ADELE B., Lang. Lab Dir., Univ. of Maine, 96 Falmouth St., Portland, Me. 04103

HERRERA, LAZARO, 1424 W. Paces Ferry Road, N. W., Atlanta, Georgia 30327

HERTJE, ARVELLA, Dir. Lang. Lab, St. Ambrose College, Davenport, lowa 52803

HEUERMAN, SHARON E., Woodstock Community H. S., Woodstock, III. 60098

HEWSON, DR. J., Memorial University of Newfoundland, St. John's Newfoundland, CANADA

HICKMAN, CHARLES H. JR., 4025 South 8th St., St. Petersburg, Fla. 33705

HICKS, DAVID B., Hd. Mod. Lang. Dept., The Pingry School, North Ave., Hillside, N. J. 07207

HIGH SCHOOL RESEARCH CENTER, Felsooktatasi Pedagogiai, Kutatokozpont, Budapest. Hungary VIII Rigo utca 16

HILl, REV. FATHER SEBASTIAN, Chmn. Mod. Lang., Father Judge Seminary, Monroe, Va. 24574

HILTS, MRS. MARGARETE, Loma Linda Univ., Riverside, Cal. 92505

HINKLE, RUTH C., 106 Buffalo Street, Elkins, West Virginia 26241

HNNTLIAN, MRS. S., Chairman, Foreign Language Dept., Enfield Senior High School. Thompsonville, Conn. 06082 
HIRSCH, MRS. BERNICE G., Dir. Lang. Lab, Samford Univ., 800 Lakeshore Dr., Birmingbam. Ala. 35209

HOCKING, ELTON, Prof. Mod. Lang. Educa., Purdue Univ., Coulter Hall. Lafayette, Ind. 47907

HOLlAND, REUBEN W., University of Chattanooga, Chattanooga, Tennessee 37403

HOOT, JAY, German Inste., Northland Colloge, Ashland, Wis. 54806

HOPKINS, AIRED H., Jr. French and English Instr., Middlefield Memorial Junior H. S.. 80 Strong Rd., S. Windsor, Conn. 06074

HOULIHAN, ROBERT, Director, English Language Institute, Tokyo, 15-9 3-Chome, Higashi Ikebukuro, Toshima-Ku, Tokyo, JAPAN

HOWELL, NORA C., Dir. Lang. Lab, Appalachian State Univ., Boone, N. C. 28607

HOWLING, ROBERT T., Chmn. English and Humanities, New Haven College, New Haven. Conn. 06505

HUBBARD, DR. LOUISE J., Chmn. Lang. Dept., District of Columbus Teachers College, 11th and Harvard Streets, N. W., Washington, D. C. 20009

HUBERT, BALCAEN, 500 Dysart Rd., Winnepeg 19, Manitoba CANADA

HUGHES, EUGENE E., Chmn. Division of Lang. Arts, Southwestern State College, Weather. ford, Oklahoma 73096

HUGHES, WILLIAM H., College of Southern Idaho, P. O. Box 1238, Twin Falls, Idaho 83301

HULL, ALEX P. JR., Assoc. Professor Foreign Language, Director Languages Laboratory. Texas Technological College, P. O. Box 4385, Lubbock, Texas 79409

HUTCHINSON, DR. JOSEPH C., Academic Advisor, Hd. Defense Language Institute, 302 G. St. SW, Washington, D. C. 20024

$$
-1 \text { - }
$$

IANNUZZI, Prof. D. A., Chmn. Mod. Lang. Dept., P. O. Box 164. Niagara Univ., N. Y. 14109

IBBOTSON, A., Dir. Mod. Lang. Audio-Visual Center, Queen's Univ., Kingston, Ontatio CANADA

INGERSOLL, J., Teacher, Senior H. S., Grand Rapids, Mich. 49500

IOUP, GEORGETTE L., Student, 402 W. 22nd St. N. 3F, New York, N. Y. 10011

$$
\text { - J- }
$$

JABlON, DR. KeNNETH, Professor and Head of MFL, Frostburg State College, Frostburg. Maryland 21532

JACKSON, GORDON, Instr., Whitman College, Walta Walla, Washington 99362

JACKSON, JAMES W., LL Dir., Western Michigan Univ., Dept. of Lang., Kalamazoo, Mich. 49001

JACKSON, DR. RICHARD, 3323 Belvedere Rd., West Palm Beach, Fla. 33401

JACQUES, FERNAND, Instr., Lakewood H. S., 13429 Emerson Ave., Lakewood, Ohio 44107

JALLING, HANS, Dir., LL Research Project, Box 6404, 11382 Stockholm, SWEDEN

JARLETT, FRANCIS G., Dir. Lang. Lab., Central Connecticut State College, New Britain. Ct 06050

JOHNGREN, RUTH, Dir. For. Lang. Dept., Norwood Senior High, Norwood, Mass. 02062

JONES, R. J., Univ. of Toronto, The College of Education, 371 Bloor St. W., Toronto, Ontasio CANADA

JUAN, SISTER M., Chmn., Fr. Dept., Regis College, 235 Wellesley St., Weston, Ma 02193

$$
\text { - K - }
$$

KADAR, DR. G., NAPA College, Dept. of FL, Napa, California 94558

KAWARABAYASHI, YUSUKE, Dir. LL, University of the Pacific, Stockton, Cal. 95204

KAY, MR. MICHAEL L., Dir. Ling. Lang. Training Pro., York Univ., 4700 Keele St., Downs. view, Toronto, Ontario CANADA

KEHOE, DENNIS. Lang. Lab. Dis., WSU-Whitewater, Whitewater, Wis. $\mathbf{3 3 1 9 0}$

KEK, DR. ANNA DALE, Chmn. Lang. Dept., Davis and Elkins College, Elkins, w. V2. 26241 
KRLIER, J. P., Albion College, Albion, Mich. 49224

KELIINGER, MRS. CESI, Lab. Dir., Wilson College, Chambersburg, Penn 17201

KEMP, MR. LINWOOD, Westfield State College, Westfield, Mass 01085

KEMPRECOS, JOSEPH, LLD, Rumson Fair Haven Regional High School, Rumson, New Jersey 07760

KeNT, SISTER MARY KATHERINE, Chmn. MFL Dept., Saint Xavier College, 103rd. and Central Park Ave., Chicago, Ill 60655

KERR, MRS. CHARLOTTE, Supv. Lang. Lab, Occidental College, 1600 Campus Rd., Los Angeles, Cal 90041

KIEKEL, R. D., Ass't Prof., Dept. of Mod. Lang., Oregon State Univ., Corvallis, Oregon 97331 KIMMEL, Dr. ARTHUR S., Assoc. Prof., Salem State College, Salem, Ma 01970

KIZER, WALLACE, Baptist College at Charleston, P. O. Box 10087, Charleston, S. C. 29411

KLEINSASSER, J. A., Sioux Fall College, Sioux Falls, South Dakota 57101

KLESOWSKI, THOMAS M., Montville H. S., Oakdalc, Conn 06370

KOPP, DR. LaMarn W., 138 Sparks Bldg., Univ. Park. Penn. 16802

KNUDSON,, JOHN K., Univ, of Pittsburgh, Dept. of General Ling., Pirtsburgh, Penn. 15213

KOEHLeR, GeORGE E., Lang. Lab. Dir., St. Procopius College, Lisle, III 60532

KOPPENHAVER, JOHN, Hd. Sp. Dept., Hesston College, Hesston, Ks. 67062

KRAIL, JACK B., Dir. Lang. Lab, Norfolk State College, 2401 Corprew Ave., Norfolk, Va. 23502

KRUSE, E. L. JR., Lecturer in Educ. and Mod. Lang., Canisius College Buffalo, N. Y. 14208

KULTURA, Newspaper Import Company Egyetemi Konyvtar, POB 483, (Scientific Library, U. of Budapest), Budapest, HUNGARY

\section{$-\mathbf{L}-$}

LABORATORY DIRECTORS, Board of Education, 97 Maple St., Summit, N. J. 07901

LANGLeY, TASSIE REE, Assistant Professor of English, Atlantic Christian College, Dept. of English, Wilson, N. C. 27893

LANGUAGE LABORATORY, Launrentian Univ., Sudbury Univ., Sudbury, Ontario CANADA

LANGUAGE LABORATORY DIRECTOR, Brevard College, Brevard, N. C., 28712

LANGUAGE LABORATORY DIRECTOR, Univ, of Connecticut, Storrs, Conn. 06268

LANGUAGE LABORATORY DIRECTOR, The College of Wooster, Wooster, Ohio 44691

LABROSSE, ANDRE, Language Dir., Windsor Locks Jr. H. S., Windsor Locks, Conn 06096

LACEFIELD, ARCH S., Sr. Instr., College Library-Univ. of Ky., Henderson Community College. Henderson, Ky 42420

LACKAU, PAUl, Brigham Young Univ., Lang. Lab 215 McKay Bldg., Provo, Ut 84601

LAFOREST, SISTeR elizabeTh, Chmn. Dept. of FL; Prof. of Fr., Mercy College of Detroit, 8200 West Outer Drive, Detroit, Mich. 48219

LANGe, MR. DAle L., Asst. Prof., 152-A Peik Hall, Univ. of Minn., Minneapolis, Minn. 55455

LANGE, JOHN B., Assoc. Prof., St. Benedict's College, 526 Division, Archison, Kas. 66002

LAPP, DR. DONALD, Aruora College, Aruora, III. 60507

LARKIN, JAMES B., Dir. Lang. Lab, Box 33, Coe College, Cedar Rapids, Ia 52402

LARSON, MRS. ELINOR C., Lab Dir., Scotch Plains-Fanwood H. S., Westfield Rd., Scotch Plains, N. J. 07076

LASATER, HelgA D., Teaching Aide, Foreign Language, Monterey Penisula College, 491 Hawthorne Street, No. 8, Monterey, California 93940

LASHUA, MRS. MARGARET, Dir. For. Lang., Glastonbury Public Schools, Gideon Welles Jr. H. S., Glastonbury, Conn 06033

LAWLOR, JOSEPH, P., Dir. Lang. Lab, Lake Forest H. S., 1285 North McKinley Rd., Lake Forest, III. 60045

LAY, AMADO M., Asst. Prof. of Sp., Gustavus Adolphus College, Box 1413. St. Peter, Minn 56082

LEAHY, S. PETER, Wisconsin State Univ..St. Point, Stevens Point, Wis 54481

LEE, DAVID, Chmn., For. Lang. Dept., Fisk Univ., Nashville, Tenn. 37203 
LEE, MARIE LORRAINE, Fr. Dept., Far Hills Country Day School, Far Hills, N. J. 07931

LEIGHTON, CHARLES, Chmn. Dept. of Sp., Univ, of New Hampshire, Durham, N. H. 03824

LEWIS, MRS. MARSHA, Tompkins-Cortland Community College, 175 Main Street, Groton, N. Y. 13073

LEONE, ARTHUR A., 2593 Shelly Dr., Indiana, Penn. 15701

LEVINSON, MRS. B., Brooklyn College-City Univ., Bedford Ave. and Ave. H, Brooklyn. N. Y. 11210

LIBRARIAN, Auckland Teachers' College. 74 Epsom Avenue, Auckland S. E. 3, New Zealand LIEBIG, GLEN, LL. Director, Bryan College, Dayton, Tennessee 37321

LINDBERG, R. H., Chmn. Lang. Dept., Gov. Dummer Academy, Byfield, Ma 01922

LINDNER, DR. LUIS, Stephens College, Columbia, Mo 65201

LIST, DR. EDGAR A., Chmn. Dept. of For. Lang., Fresno State College, Fresno, Cal. 93726

LOMBARDO, S. JOSEPHINE, Rosary Hill College, 4380 Main St., Buffalo, N. Y. 14226

LOOMIS, MRS. RUTH, F. L. Curriculum Associate, Madison Memorial H. S., 201 S. Gammon Rd., Madison, Wis. 53705

LORE, DR. A. G., Dir. Lang. Lab., Univ, of N. C., 105 Dry Hall, Chapel Hill, N. C. 27514

LOUBERT, SISTER ELAINE, College of St. Scholastica, Duluth, Minn. 55811

LUBIC, MICHAEL K., Miles College, Birmingham, Alabama 35208

LUBS, MARGARET, Professor, Armstrong State College, Savannah, Ga 31406

LUCAS, BOYCE M., Dir. Lang. Lab, Whitehall H. S., Whitehall, Mich. 49461

LYNCH, ANNA M., Dir. Lang. Lab, Trinity College, Michigan Ave., Washington, D. C. 20900

LYTLE. MRS. B. G.. Assistant Lab. Tech.. Onondago Community College, 700 East Water Street, Syracuse, N. Y. 13210

LYTTON, MRS. VEGA, Lang. Lab. Dir., Mary Baldwin College, Staunton, Va 24401

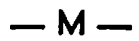

MACINKO, JOHN, Dir. Lang. Lab, Univ. of Colorado, 303 Old Main, Boulder, Colorado 80302

MADELEINE, SISTER M., College of Notre Dame of Md., Baltimore, Md. 21210

MAESTAS, M. M., 5570 Winchelsea Drive, Normandy, Mo 63121

MAGDALEN, SISTER MARIE, O. P., Rosary College, River Forest, Illinois 60305

MAHLER, MICHAEL, 8001 Natural Bridge, Se. Louis, Mo 63121

MANDEL, E. JULES, Coord. Supv. Techn. UCLA, 20918 Calimali Rd., Woodland Hills, Cat. 91364

MANN, G. THOMAS, Graceland College, Lamoni, Iowa 50140

MAPA, SISTER M. V., Lang. Lab, Dir., Lone Mt., San Francisco, Cal. 94118

MANNING, SISTER MARGARET, Dir, Lang. Lab, Notre Dame College of Staten Island, 300 Howard Ave., Staten Island, N. Y. 10301

MANOLIS, JOHN C., L.L Dir., Mary Washington Col. of U of Vir., P. O. Box 1126, College Station, Fredericksburg, Vir. 22401

MANWELL, RACHEL, Lang. Lab. Asst., Univ. of Illinois, 214 Lincoln Mall, Urbana, IIl. 61801

MARKLE, DOROTHY, Coord. FL, Newark State College, Morris Ave., Union, N. J. 07083

MARShALL, ROBERT T., Chmn., Dept. of Lang. and Ling., Mt. St. Mary's R. D. 1, Emmitsburg, Md 21727

MARSHALL, WILliam H., Asst. Prof, of Lang., Univ. of Maine, Augusta, Maine 04300

MARTIN, MR. WILLARD M., Acting Dir. of Lang. Lab, Penn. State Univ., 6 Sparks Penn State Univ., University Park, Penn 16802

MARTINEZ, MR. CARLOS CORTES, Dir. Lang. Lab, Deusto Univ., Apartado De Correos No. 1147, Bilbao-Vizcaya, SPAIN

MARTINEZ, ROGELIA, Dir. Lang. Lab. 914 Gaylemont Ct., Decator, Ga. 30033

MARTINEZ, DR. R. V., Chmn. Sp. Dept., Franklin and Marshall College, Lancaster, Penn. 17601

MATRANGA, EDWARD C., Supv. of FL, Stratford Town Schools, Stratford, Ct 06497

MATTHEWS, HELENE, Dir. of LL, 89 Collins Ave., Sayville, N. Y. 11782 
MARIE SISTER THERESE, Chmn. FL Dept., Good Counsel College, 32 North Broadway, White Plains, N. Y. 10603

MARY, SISTER JeAN, O. P., Chmn. Fl Dept., Barty College, Mizmi, Fla. 33161

MAXWELL. WILliaM, Head Language Dept., Tabor Academy, Box 372, Marion, Ma. 02738

McCULlOUGH, MR. J. T., Sacramento State College, Sacramento, Cal. 95819

MCCUNE, JERRY, Indiana Univ., P. O. Box 1125, Bloomington, Ind. 47401

MCGETTRICK, MISS HELEN, 115 St. Stephen Street, Boston Massachusetts 02115

McKeNNA, J., Language Laboratory Director, College of the Holy Ctoss, Worcester, Maine 01610

McKeNNA, MRS., Secretary, Hyde School, Bath, Maine 04530

MclaNe, JEAN, Assistant Professor, McPherson College, McPherson, Kansas 67460

MeMUllaN, JOHN, Head, Language Department, St. Georges School, Newport, Rhode Island 92840

McNAMARA, MRS. M. J., Supv.. Language Lab., Arts, Bldg., Douglass College, New Brunswick, New Jersey 08903

MEIER, MR. WALTER, Seebacherstrasse 80, Zurich, Switzerland

MERCEDES, SISTER IRMA, Instruetor, Chestnut Hill College, Philadelphia, Pennsylvania 19118

MerkensteijM, E. C. VAN, 320 Logan Hall, University of Pennsylvania, Philadelphia, Pennsylvania 19104

MESS, MARY JANE, Ottawa Township High School, Ottawa, Illinois 61350

MESSNER, C. A., Associate Professor of Modern Languages, Carteton College, Northfield, Minnesota 55057

METFORD, DR. JACQUES, Director of Language Laboratories, University College, UWO. London, Ontario, Canada

METTLER, DONALD G., Language Laboratory Director, Edison Township High Scbool, South Colton Road, Edison. New Jersey 08817

MEYER, E. O., Hamlin University, St. Paul, Minnesota $\$ \$ 101$

MICKO, MICHAEL, Director, Language Laboratory, PMC Colleges, 818 E. 18th Street, Chester. Pennsylvania 19013

MIDDLETON, R. T., Director, Language Laboratory, Tougaloo College, Tougaloo, Mississippi 39174

MIELE, MR. HARRY, Language Department, 340 Frederick Street, Cumberland, Maryland 21502

MIKESELL, NORMAN, Indiana University, Ballantine Hall, Room 120, Bloomington, Indjana 47401

MILES, DR. E. KENNETH, Professos of German, 214 Litele Hall, University of Maine, Orono, Maine 04473

MILLER, EUGENE, Council of Modern Languages, 227 Crosby Hall. State University of New York at Buffalo, 250 Winspear Ave., Buffalo, N. Y. 14214

MILLER. J. DALE, Associate Professor of French and Italian, 360 McKay Building, Brigham Young University, Provo, Utah 84601

MILLER, MRS. MARTHA R., Secretary to Director, Harvard University Modern Language Center, Boylston Hall G-3, Cambridge, Massachusetts, 02138

MIILER, ROBERT C., Chairman, Modern Language Department, The Thacher Sebool, Ojai. California 93023

MIILER, SHELBY C., 128 Pearl Street, Cambridge, Massachusetts, 02139

MILLMAN, MRS. WM. B., Director, Rider College, Trenton, New Jersey 08602

MILLS, DR. DOROTHY HURST, Department of Foreign Language Laboratory, Chapman College, Orange, California 92666

MIREL, BERNARD. Director Language Laboratory, 6738 Magoun, Hammond, Indiana 46324

MITROVICH, MIRCo M., Ph.D., Associate Professor, Muskingum College, Johnson Hall $104 / 105$ New Concord, Ohio 43762

MTTTAG, MARLIN M., 910 West Downer Place, Aurora, Illinois 60506

MOEN, MRS. MARY E., Instructor, Anokz Ramsey State Junior College, 701 Mr. Curve Blvd.. St. Paul, Minnesota $\$ 5116$

MOISSIY, TAMARA S., Director, Language Laboratory, 10 Mount Pleasant Street, Hyde Park. Maine 02136

MOORE, MRS. MERRIAM, Department Chairman of Foreign Languzges. Ridgefield High School, P. O. Box 353, Ridgefield, Connecticut 06877 
MOORE, DR. MICHAEL J., Specialist FL Education, San Diego Board of Education, 4100 Normal Avenue, San Diego, California 92103

MONSON. EDITH L., Chairman, Foreign Language Department, Georgetown College, Georgerown, Kentucky 40324

MONTAGUe, COLONEL H. P., 196 Beach 128th Street, Belle Harbos, New Yosk 11694

MORAN, SISTER M. CATHERINE, O. P., Chairman, Department of Foreign Languages, Edgewood College, Madison, Wisconsin 53711

MORENO, DR. ANTONIO, 752 Lockhart Street, Washington, Pennsylvania 15301

MORGAN, MRS. MARGARET, Director, Language Laboratory, Sacred Heart Dominican College, 2401 East Holcombe, Houston, Texas 77021

MORRISON, MR. R., Chairman, Modern Languages, Southern Missionary College, P. O. Box 475. Collegedale, Tennessee 37315

MOUTAIN, ClifF, Director, Foreign Languages, Mesa College, 1120 North Aveaue, Grand Junction, Colorado 81501

MULHOLLAND, CHARLES V., Language Laboratory Supervisor, University of Rhode Island, Department of Languages. Kingston, Rhode Island 02881

MURDOCH, MRS. CHRISTINE, Instructor, Southern Missionary College, Box 422, Collegedale, Tennessee 37315

MURPHY, HAROLD T., Associate Professor, Director of Language Laboratory, P. O. Box 238, Marshall University, Huntington, West Virginia 25701

\section{$-\mathbf{N}-$}

NABERS, JAMES E., Southem Illinois University, Carbondale, Illinois 62901

NACHREINEI, E., Assoc. Prof. German, Andrews University, Berrien Springs, Michigan 49104

NARVAEZ, LEON ORLAN, Teaching Asst., Language Laboratory, University of Minnesota, Minneapolis, 2030 Brewster St., Apt. 13. St. Paul, Minnesota 55108

NEARHOFF, ORRIN, 2745 Bennett Avenue Des Moines, Iowa 50310

NEHER, WILliAM R., Head of Language Dept., 4200 W. River Road, Minneapolis, Minnesota 55406

NEWMAN, BERNARD, Teacher in Charge of Language Laboratory, Bayside High School, 32-24 Corp. Kennedy St.. Bayside, New York 1

NEWMAN, JAMES L. V., Simmons College, 300 The Fenway, Boston, Massachusetts 02115

NEWMAN, RICHARD W., Chairman, Foreign Language Dept., 625 Huntington Ave., Boston, Mass. 02125

NEWMARK, LEONARD, Chairman, Dept. of Linguistics, University of California at San Diego, La Jolla, California 92037

NICHOLSON, CHARLES E., Consultant, MFL, 120 E. 10th, Topeka, Kansas 66612

NIXON, RUTH A., Chairman FL, Wisconsin State University, La Crosse, Wisconsin 54601

NORWICH, THEODORA, Language Laboratory Director, Western College for Women, Oxford, Ohio 45056

NOVAK, PROF, RICHARD, Director of Language Laboratory, Concordia College, 171 White Plains Road, Bronxville, New York 10708

NOVAK, MR. WITOLD, Director, Language Laboratory and A.V Center, Monmouth College, Monmouth, Illinojs 61462

NUFFER, J. STANLEY, Director of Language Laboratory, Portland State College, Box 731 Foreign Language Lab, Portland, Oregon 97207

NUNN, ROBERT R., Director, Bowdoin College, Brunswick, Maine 04011

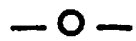

OBRATH, KARL W., Director, Language Lab, University of Cincinnati, 700 Riddle Road No. 202, Cincinnati, Ohio 45220

O'CONNELI, MR. THOMAS F., Smith College, Wright Hall 7, Northbampron, Massachusetts 01060

OJEDA, MRS. AMPARO B., Language Laboratory Dept., Loyola University, North Sheridan Road, Chicago, Illinois 60626 
OKLAHOMA BAPTIST UNIVERSITY, Modern Language Laboratory, Shawnee, Oklahoma 74801

OKSENHALT, DR. SVEIN, Head, Department of Foreign Languages, Eastern Montana College. Billings, Montana 59101

OLSEN. IRVING S., Director Instructional Materials, Valparaiso University. MFL Room 147. Valparaiso. Indiana 46383

O'NEIL, WILliAM J., Jr.. Chief Technician. New York University, 64085 84th Street, Rego Park, N. Y. 11379

OTTO, DR. VIRGINIA, Chairman of Foreign Language Dept. Nazaretb College of Rochester. 4245 East Avenue, Rochester. New York 14610

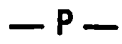

PADEN, TERRENCE, Director, Language Laboratory, Upper lowa College, P. O. Box 383. Fayette, Iowa 52142

PAQUETTE, ClAUDE A., Language Laboratory Director, Foreign Language Department, University of Bridgeport,. Bridgeport, Connecticut 06602

PAQUETTE, F. A., Executive Secretary, ACTFL, 62 Fifth Ave., New York, New York 10011

PARSONS, MRS. ElaINE, Language Laboratory, Green Mountain College, Poultney, Vermont 05764

PATINO, MR. ARMANDO, Defense Language Institute, East Coast Branch, U. S. Naval Station, Anacostia Annex, Washington, D. C. 20390

PATRON, GLORIA A., Director, Language Laboratory, Louisiana State University, Lakefront, New Orleans, Louisiana 70122

PAUCHET, MRS. JACQUELINE, Laboratory Supervisor, Knotville College, Route 4, Ruggles Ferry Road, Knoxville, Tennessec 37914

PAUCKe, E., Dalton Hall, Bryn Mawr College, Bryn Mawr, Pennsylvania 19010

PAUL, MRS. CHRISTA E., Language Laboratory Director, Philadelphia College of Pharmacy and Science, 43rd Strect and Kingsessing Ave., Philadelphia, Pennsylvania 19147

PAYNE, EDWARD W., P. E., Supervisor, Language Laboratory, Malone College, 515 25th Street N. W.. Canton, Ohio 44709

PEAK, J. HUNTER, Auburn University, Mell Hall 205, Auburn, Alabama 36830

PEARSON, ROSE D., Supervisor, Language Laboratory, 1540 Unquowa Road, Fairfield, Connecticut 06430

PELOQUIN, PAUL D., Chicopee Comprehensive High School, Rolf Avenue, Chicopee Falls, Massachusetts 01020

PENCHOEN, THOMAS, Assistant Professor of Berber Languages, Royce Hall 302, UCLA, 405 Hilgard Ave., Los Angeles, Cal. 90024

PENNEY, GEORGE J., High School, 869 Forbes Street, East Hattford, Connecticut 06108

PENTA, RICHARD M., 38 Normandy Road, Lexington, Massachusetts 02173

PEREZ-SABIDO, J., 4106 Windward Drive, Lansing. Michigan 48910

PETERSON, PAUL W., Director, Department of Modern Languages. Gannon College, Erie. Pennsylvania 16501

PETRIE, THE REVEREND R., A.V Coordinator, St. Joseph Seminary, Callicoon, New York 12723

PHILIIPPON, CHRISTIAN, Hostal Horche-Gaztambide 59, Madrid 2, Spain

PICCO, MRS. ELSIE, Supervisor, Language Laboratory, Bennington College, Bennington, Vermont 05201

PILKANIS, WILliaM J., Director, Language Laboratory, Rbode Island Junios College, 199 Promenade Street, Providence, Rhode Island 02908

PIMSLEUR, DR. PAUL, Director, The Listening Center, Obio State University, 164 West 17th Avenue, Columbus, Ohio 43210

PIOLA, GLADYS, Director, Language Laboratory, New Canaan High School, New Canaan, Connecticut 06840

POIRIER, DR. ROGER A., Director, Language Laboratory, Union College, Springfield Avenue. Crawford, New Jersey 07016 
POISSANT, HERVEY, St. Francis College, Pool Road, Biddeford, Maine 04005

PORTERA, JOHN, Lab Director. San Fernando Valley State College, Foreign Language Department, 18111 Nordhoff Street, Northridge, Cal. 91324

PRADES, B. J., Spanish Instructor; Director, Language Laboratory, Foreign Language Department, 444 Armstrong Hall, West Virginia University, Morgantown, West Virginia 26506

PRESTON, DR. F. L., Associate Professor, Denison University, Box 267, Granville, Ohio 43023

PROCOPIO, DOMENIC R., Chairman, Department of Foreign Languages, Lowell State College, Lowell, Massachusetes 01854

PROTASE, SISTER M., Associate Professor of French, College of St. Francis, 500 Wilcox Street, Joliet, Illinois 60435

PURICELLI, SISTER AGNES, C. S. J., Instructor, Fontbonne College 6800 Wydown Boulevard, St. Louis, Missouri 63105

\section{- R -}

RACINE, DANIEL, Howard University, Washington, D. C. 20009

RAGLE, GORDON, Assistant Professor of Spanish, 5225 Troost Avenue, Kansas City, Missouri 64110

RAICHLE, LOUIS P., Professor, Staten Island Community College, Ocean Terrace, Staten Island, New York 10301

RECEIVING DEPARTMENT, Sonoma State College, 1801 East Cotati Avenue, Rohnert Park, California 94928

REDDEN, JAMES E., Associate Professor of English, Southern Illinois University, Department of English, Carbondale, Ill. 62901

REESE, MR. WINSTON J., Director, Language Laboratory, University of Iowa, 125 Schaeffer Hall, Iowa City, Iowa $\mathbf{5 2 2 4 0}$

REEVES, DONA, Chairman, Modern Language Department, Southwest Texas State College, 1101 Robert E. Lee Road. Austin, Texas 78666

REGINA MARIE, SISTER, O. P., Siena Heights College, Adrian, Michigan 49221

REID, MISS CONSTANCE L., Gettysburg College, Gettysburg, Pennsylvania 17325

REID, MRS. FRANCES, Supervisor, Language Laboratory, Davidson College, Davidson, North Carolina 28036

REID, MRS. GHISLAINE, Director of French, Metropolitan Separate School Building, 146 Laird Drive. Toronto 17, Ontario, Canada

REID, J. RICHARD, Department Chairman of Romance Languages, Clark University, Worcester, Massachusetts 01610

REITINGER, DR. GOTTFRIED, Director of Studies, Universitat Innsbruck, Institut fur Delmentscherausbildung, Fischnalerstrasse 4, 6020 Innsbruck, Austria

RHODE, SISTER ELIZABETH, Chairman Modern Language Department, Alverno College, 3401 South 39th Street, Milwaukee, Wisconsin 53215

RICHARDSON, SISTER JANET, Chairman, Foreign Language Department, Englewood Cliffs College, Englewood Cliffs, New Jersey 07632

RIEP, A. R.. Chmn. Dept. of Mod. Lang., Wartburg College, Waverley, lowa 50677

RIUSECH, ALBert, Asse. Prof. of Languages, Ouachita Baptist University, Box 739, Arka. delphia. Arkansas 71923

RIVERS, DR. W. M., Monash University, Clayton Victoria 3168, Australia

ROBINSON, MRS. FRANCES, Head, Lang. Dept., Newington High School, 131 Cedar Street, Newington, Conn. 06111

RODGERS, MRS. MARY LUNN, Chairman, Dept. For. Lang., Box 643, H-S Station, Abilene, Texas 70601

ROSARIA, SISTER MARY, 3900 Peterson Avenue, Chicago, Illinois 60645

ROSARIO, SISTER, Chairman, Department of Foreign Languages, College of the Holy Names. 3500 Mountain Boulevard, Oakland, California 94619

ROSE TERESA AMOR, SISTER, Chairman, Spanish Department, Molloy Catholic College, Rockville Centre, New York 11570

ROSENDA, SISTER, Director, Modern Languages, Language Laboratory Coordinator, Regis College, Weston, Massachusetts 02193

ROSELDA, S. M., Head, Modern Language Department, Notre Dame High School, 9th and Plum Streets, Breese, Illinois 62230 
ROSENFIELD, MRS. PATRICIA B., Director, Language Laboratory. Newton Junior College, Washington Park, Newronville, Massachusetts 02160

ROSS, CHARLES E., Teaching Assistant, University of Southern California, 10711 Rose Avenue, Los Angeles, California 90034

RUGGLES, GORDON W., Director, Ashland College, College Ave., Ashland, Ohio 44805

RUPPRECHT, THE REVEREND MELVIN, Laboratory Director, St. Vincent College, Latrobe, Pennsylvania 15650

RUSCH, NORMA, Director. Language Laboratory, Lakeland Union High School. Minocque, Wisconsin 54548

RYAN, MRS. CAROLE ANN, Assistant Professor of French, Illinois College, Jacksonville, Illinois 62650

RYBERG, LILlIAN, 1480 N. Snelling, St. Paul, Minnesota 53101

\section{$-S-$}

SACHER, CECIL, Chairman Foreign Languages. Niles Township High School North, 9800 Lawler, Skokie, Illinois 60076

SADberRY, LONNIE, Professor of Spanish, Prairic View A and M College, Prairic View, Texas 77445

SALAS, SAMUEL A., Director Language Laboratory, 520 Lone Pine Road, Bloomfield Hills, Michigan 48013

SAMANIEGO, F. A., Instructor, Utah State University, Department of Language, Iagan, Utah 84321

SAMPON, VICTOR, Wayland Academy, Beaver Dam, Wis. 53916

SANCHEZ, MRS. MARY, Supervisor, Foreign Languages, 1960 East Druid Road, P. O. Box 4688, Clearwater, Florida 33518

SAWYER, J. O., University of California, Berkeley, California 94720

SCARTH, MARGARET, Lecturet in Spanish, Erindale College, University of Toronto, Toronto 5. Ontario, Canada

SCHAEFER, MRS. U. M., Supervisor, Language Laboratory, Connecticut College, Box 1524,

New London, Connecticue

SCHAUBACHER, Supervisor, Lang. Lab., Shipley School, Yarrow Street, Bryan Mawr, Pa. 19010

SCHMOLI, RONAID B., Assistant Professor of German, Asheville-Baltimore College, Asheville, North Carolina 28801

SCHOLTIS, EBERHARD, Lang. Lab. Dir., Plattsburgh State University College, Plattsburgh, N. Y. 12901

SCHULTZ, DAVID, Supervisor Language Laboratory, State University of New York, Maritime College, Fort Schuyler, Bronx, New York 10465

SCHULTZ, GEORGE F., Associate Professor of Modern Languages, Central State University, 109 N. Washington Strect, Columbia City, Indiana 46725

SCHW ANK, DENNIS M., Coordinator Language Laboratory, University of Minnesota, Morris, Minnesota 56267

SCHWENKER, D., 230 Edinburgh Road S., Guelph, Ontario, Canada

SCOTT, S. N., Linguistics Instructor, Kirkland College, Clinton, New York 13323

SENG, MARK W., Assistant Prof., University of Texas, 300 E. Riverside, No. 111, Austin, Texas 78704

SERAFINO, ROBERT P., Supervisor of For. Lang., New Haven, Ct. 06525

SHAPIRO, MRS. MARY, Bridgewater State College. Bridgewater. Massachusetts 02324

SHARPE, P. B., 132 Main Street, Franklin, Massachusetts 02038

SHAW, DR. ARACELIS G., Chairman, Romance Language Department, Columbia College, Columbia, South Carolina 29203

SHEEHAN, JOSEPH H., Georgetown University, Washington, D. C. 20000

SHerraRD, DAVID A., Chief Audio-Visual Branch, Defense Language Institute East Coast, U. S. Naval Station, Anacostia Anaex, Washington, D. C. 20390

SHIREY, MARY M., 2419 Longview Avenue SW. Roanoke, Virginia 24014 
SHREFFLER, E. H., Director, Language Laboratory, Austin College, Box 1239, Sherman, Teres 75091

SIMMONS' HOW ARD L., Lab Director, 38 Plaza South, Apt. 705, St. Louis, Missouri 63103

SIMmONS, S. J. JOSEPH, Head, Modern Languages, Geosgetown Preparatory School, 1225 Otis Street, N. E., Washington, D. C. 20017

SIREVAAG, JOHN, Head, German Department, Augustana College, Rock Island, Illinois 61202

SISTER JOAN OF ARC, O. P., Rosary High School, 8551 Greenfield Avenue, Detroit, Michigan 48228

SKANSE, PETER 1. R., Director, Language Laboratory, Wheaton College, 425 East Oak Avenue, Wheaton, Illinois 60187

SKW AREK, RUTH, Instructor, Muskegon County Community College, 15088 W. Leonard Road, Spring Lake, Michigan 49456

SMALL, SOL, Tecbnical Director, Language Laboratoty, C. W. Post College, Brookville, New York 11548

SMITH, PROFESSOR NELLENE. Director Instructional Media, Monterey Institute of Foreign Studies, 425 Van Buren, Montercy, California 93942

SMITH, W. FLINT, Purdue University, Lafayette, Indiana 47907

SMITH, DR. PHILIP, Learning and Research Center, High and Rosedale Avenue, West Chester, Pennsylvania 19380

SNYDER, SISTER JANE DE CHANTAL, 6800 Wydown Boulevard, St. Louis, Missouri 63105

SOKOLOW, HENRY N., Director. Language Labotatories, Santa Monica City College. Santa Monica, California 90401

SPEAR, FREDERICK A., Associate Professor of French, Skirmore College, Saratoga Springs, New York 12866

SPETRINO, ANTHONY, Chairman, For. Lang. Dept., Bassick High School, 1181 Fairfield Ave., Bridgeport, Conn. 06605

SPINKS, O. R., Director, Suny at Albany, Humanities B-16, Albany, New York 12203

SODERMAN, LEONARDO, Language Laboratory Director, University of Concepcion, Casilla 2307, Concepeion, Chile.

SPENCE, PATRICK, Assistant Director Language Laboratory, Baptist College, P. O. Box 10087. Charleston, South Carolina 29411

STACK, E. M., Professor, 3925 Arrow Drive, Raleigh, North Carolina 27609

STADT, MRS. B. W., Rollins College, Box 119, Winter Park, Florida 32789

STEINBERG, ADRIAN L., Ditector, Foreign Language Laboratory, Temple University, 3rd Floor, College Hall, Philadelphia, Pennsylvania 19122

STEWART, ANDREW T., Head, Foreign Language Department, Cardigan Mountain School, Canaa, New Hampshire 03741

STEV ENS. EDITH, 1211 4th Avenue S., Moorhead, Minnesota 56560

STITES, KENNETH D., Language Laboratory Director, Wilmington College, Wilmington, North Carolina 28401

STONER, MRS. MARJORIE M., Executive Secretary, Language Center, Soutbwestern University at Memphis, Memphis, Tennessee 38112

SUAREZ, J. DAVID. Instructor Modern Languages, 19 Blackthorn Road, Framingham, Massachusetts 01701

SWANSON, MR. S. A., R. R. 1, Box 135, Omro, Wisconsin 54963

SWIFT, MRS. MARTHA H., Morgan Park Academy, 2153 West 111 th Street. Chicago, Illinois 60643

\section{$-\mathrm{T}-$}

TANNER, MRS. J. M., Director, Romance Language Department, University of Virginiz, 302 Cabell Hall, Charlottesville, Virginia 22903

TARAS, DR. A. F., Director Audio Laboratory Center, Ithaca College, Itbaca,. New York 14850

TAYLOR, MR. WALTER, Upsala College, East Orange, New Jersey 07019

THADDEUS, SISTER JUDE, Chairman, Spanish Department, Seton Hill College, Greensburg, Pennsylvania 15601

THARAUD, MRS. CYNTHIA J., Director Language Laboratory, 308 Philosophy Hall, Colugbia University, New York, New York 10027 
THATCHER, J., Assistant Head of Modern Language Department, Burlington Central Higb School, Box 5042, Burlington, Ontario, Canada

THEUMA, MR. JEAN R., Director Foreign Language Laboratory, University of Hawaii, Honolulu, Hawaii 96822

THOMAS, FREDERICK M., Catlson High School, 30550 West Jefferson Avenue, Gilbralter. Michigan 48173

THOMAS, JOSEPH V., Professor of Modern Lang., Austin Peay State University, Clarksville, Tenn. 37040

THOMPSON, MISS GAIL, Rall Hall, North Central College, Napervild, Illinois 60540

THORNE, E. R., Associate Professor, West Liberty State College, West Liberty, West Virginia 26074

THURMAN, MRS. GARY R., Instructor Modern Languages, Huntington College, Huntiagton, Indiana 46750

TIMONA, SISTER MARY, Director Language Laboratory, Mount Mary College, Milwaukec, Wisconsin 53222

TITLE, M. KeITH, Associate Prof.. University of Illinois, 1009 S. Westlawn, Champaign, III. 61820

TOCONITA, DR. M. J., Director Lang. Lab., St. Joseph's College, Philadelphia, Penn. 19131

TOIRAC, MRS. DOROTHY M.. Chairman, Language Department, Grace College, Winono Lake, Indiana 46590

TOLMAN, JON, Lecturer, Indiana University, Department of Spanish and Portuguese, Bloomington, Indiana $\mathbf{4 7 4 0 1}$

TOVEN, AUDUN, Instructor, Pacific Lutheran University, Tacoma, Washington 98447

TRAHAM, ROGER R., Director, Language Laboratory, Assumption College, 500 Salisbury Street, Worcester, Massachusetts 01609

TRIPP, MRS. GERTRUDE, Language Laboratory Director, Elmhurst College, 190 Prospect Avenue, Elmhurst, Illinois 60126

TULIOS, OSCAR, Texas Southmost College, 104 Greenway Drive, Brownsville, Texas 78520

TUMER, MARGUERIT J., Consultant, Dayton Board of Education, 354 Bungalow Road, Dayton, Ohio 45417

TURNER, ALBERT R., Director Language Laboratory, 1600 Dodge Avenue, Evanston, Illinois 60205

$-\mathbf{U}-$

UHRHAN, D. EVELYN, Director Language Laboratory, University of Guelph, Guelph, Ontario, Canada

UNIVERSITY OF GEORGIA, Department of Germanic and Slavic Languages, Athens, Georgia 30601

UNIVERSITY OF WESTERN KENTUCKY, Forcign Languages, Bowling Green, Kentucky 42101

\section{$-V-$}

VAlVerge, DR. LUIS J., Chmn. For. Lang. Dept., Boise State College, Box 24, Boise, Idaho 83700

VANACORE, SISTER EVANGelA, O. P., Director, Ohio Dominican College, 1216 Sunbury Road, Columbus, Ohio 43219

VAN ASSELT, MR. JAN, Head, Department of Foreign Languages, McPherson College, McPherson, Kansas 67460

VANDESTEEG, MISS MARY D., 7370 Shawnee Road, Apartment 3, Cincinnati, Ohio 45243

VAN TREeSE, GLENN J., Director Language Laboratory, Sweet Briar College, P. O. Box 18, Sweet Briar, Virginia 24595

VAN WALK GRACE R., Grants Pass High School, 522 N. E. Olive, Grants Pass, Oregon 97526

VARGAS, EDUARDO E., Assistant Professor, Missouri Western Colltge, 3402 Bel-Nor Drive, St. Joseph, Missouri 64506

VELEY, ROBERT W., Chmn. Dept. of Fl. Benson Polytechnic School, 546 N. E. 12th Ave., Portland, Ore. 97232 
VELEY, ROBERT W., Department of Foreign Languages, Mount Mary College, Milwaukce, Wisconsin $\mathbf{5 3 2 0 0}$

VERRETTE, VICTOR S., Director Language Laboratory, Grinnell College, Grinnel, Iowa 50112

VOGT, M. I., Director, Language Laboratory, University of Idaho, Ad. 322 University of Idaho, Moscow, Idaho 83843

VOJTECH, MRS. FRANK, Chairman Language Department, Anne Wright and Seminary, 827 North Tacawa Avenue, Tacoma, Washington 98403

\section{$-W-$}

WALlaCe, S. MARGareT, s11 North Center Strect, Grove City, Pennsylvania 16127

WALSH, SISTER MARIE ANDRE, IHM, Director of Language Laboratory, St. Edward University, 3001 South Congress, Austin, Texas 78704

WARE, SALLY JO, Director of Language Laboratory, Rockford College, Rockford, Illinois 61101

WARING, THOMAS R., JR., Chairman Language Department, Sewanee Military Academy. Sewanee, Tennessee 37375

WASHINGTON, MRS. ELEANOR A., Language Laboratory Director, Nettie Lee Roth High School, 727 Oak Leaf Drive, Dayton, Ohio 45408

WATSON JACK, Language Laboratory Director, Williams College, Karl E. Weston Language Center, Williamstown, Massachusetts 01267

WATSON, MR. JOHN A., Virginia Union University, Richmond, Virginia 23220

WEBB, DR. HARRY C., Audio-Visual Director, College of St. Thomas, St. Paul, Minnesota 55101

WEBBER, DONALD B., Assistant Professor Language Laboratory. The University of the South, Sewanee, Tennessee 37375

WEINKAUF, DR. ARNOLD L., Assistant Professor of Languages, Michigan Technological University, Houghton, Michigan 49931

WESTHOFF, JOHN, 100 S. 14th Str., Quincy, III. 62301

WHAN, ADELAIDE, Language Laboratory Director. University of Southern California, Department of Religion, Los Angeles, California 90007

WHEETLEY, D. W., Director, Audio-Visual Education, 194 Boylston Street, Brookline, Massachusetts 02146

WHISNANT, NORMAN, Director Language Laboratory, Furman University, Greenville, S. Carolina 29613

WHITE, RICHARD J., Director of Language Laboratory, Rich Central High School, Olympia Fields, Illinois 60461

WHITE, SIDNEY P., Head Language Department, Morgan High School, Clinton, Connecticut 06413

WHITING, PAUL, Tape Center Director, Lake Forest High School, Lake Forest, Illinois 10045

WHITINGTON, G. L., Language Laboratory Director, Cooke County Junior College, Gaines. ville, Texas 76240

WIDMAIER, KARL, Instructor Modern Languages, Atlantic Union College, South Lancaster. Massachusetts 01561

WIESE, PETER, Language Laboratory Director, Southern Connecticut State College, s01 Crescent Street, New Haven, Connecticut 06515

WILEY, RAYMOND A., Asst. Prof., LeMoyne College, Syracuse, N. Y. 13214

WILKINSON, MR. GENE, 3612 Avenue E., Kearney, Nebraska 68847

WILLECKE, DR. F. H., Chmn., For. Lang. Dept., Wagner College, Staten Island, 10301

WILLARD, PROFESSOR GEORGE, Lab Director, Union College, Springfield Ave., Craw. ford, N. J. 07016

WINN, FRANK M., Eastern Baptist College, Box 53 St. Davids Dr., Wayne, Pa. 19087

WINN, MRS. T. VADEN, Pearsall H. S., Box 1291, Pearsall, Texas 78061

WINSLOW, MARY, Ph.D., Prof. of Romance Lang., St. Mary of the Plains College, Dodge City, Kas 67801

WIPF, JOSEPH, Instr. in German, Univ. of South Dakota, Vermillion, S. D. 57069

WISNIEWSKI, RAY, Dir., A.V Services, Grand Valley State College, Allendale, Mich. 49401 
WOHR, DAVID L., Lab Asst., For. Lang. Lab, Temple University, Philadelphia, Penn 19122

WOlfram, Millard, Curric. Coord., Central Admin. Bldg., 10025 Penn. Ave., S., Bloomington, Mn. 55431

WORTHINGTON, MRS., Virginia State College, Box 428, Petersburg. Virginia 23803

WOOLSEY, JAY L., Ger. Instr., Hebron Academy, Hebron, Me 04238

\title{
$-Y-$
}

YALAMOW, DR. P., F141 Sherbrooke Street, West, Montreal, 28 CANADA

YALDEN, MRS. J. M., Lecturer, Dept. of Spanish, St. Patrick's College, Carleton Univ., 281 Echo Dr., Ottawa 7, Ontario, CANADA

YEAKEY, M. L., Department Chmn., Holland Hall School, 2640 South Birmingham Place,

- Tuls2, Ok. 74114

YOSHISHIGE, GEORGE S., Audio Visual Coord., 860 Fourth St., Pearl City, Ha 96782

YZENBAARD, JAMES, University of Kentucky, School of Letters and Languages, Lexington, Ky 40506

$$
-\mathbf{Z} \text { - }
$$

ZAANAN, DIRK VAN, 175 University Avenue, Conklin Hall, Newark, N. J, 07102

ZARR, MR. VAlOIS A., Chmn. German Dept., East High School, 1134 South 17th St. East, Salt Lake City, Utah 84108

ZEITUNGSVERTRIEBSAMT, SATAABTEILUNG, Clara-Zetkin-Strasse 62, (1004) Berlin, Germany

ZIMMERMAN, MRS. ELLEN, Lang. Lab. Dir., Thornridge H. S., Dolton, Ill. 60419

ZUMBAR, MRS. WILLIAM. Asst. Prof. Spanish, Mount Union College, Alliance, Ohio 44601 ZUZIC, PROF. DR. M., Salem College, 174 Liberty St., Salem, W. Va. 26426

NOTE: Our apologies to all members who were not listed. Some names arrived after material had been sent to the printers.

MEMBERSHIP APPLICATION for 1968-1969

Chect whether tbis is a (....) Renewal or a (....) New Member

Position

Name:

Or Title:

Name of (High School) (University) :

Circle one of the above

Address to which Newsletcer is to be sent:

Address :

City : State Zip Code:

(....) My school is paying the dues for NALLD membership; send INVOICE to:

Check for which type of membership you are applying:

(....) Regular Voting Member (USA 50 States)

(....) Regular Voting Member (Foreign \& Canadian)

(....) Regular Voting Member (Foreign desiring dir Mail)

(....) Student, Non-Voting Member

Membership runs from October to October. Please complete the above form, and return the ENTIRE SHEET to:

\author{
Sister M. Tímona. NALLD Treasurer \\ Mount Mary College \\ 2900 Nortb Menomonee River Parkway \\ Milwaukee, Wisconsin 53222
}

